Supporting Information for

\title{
Fourier Transform Infrared Spectrovoltammetry and Quantitative Modeling of Analytes in Kinetically Constrained Redox Mixtures
}

\author{
Christopher W. John, Denis A. Proshlyakov* \\ Michigan State University, Department of Chemistry \\ *Address correspondence to Denis A. Proshlyakov, Dept. of Chemistry, Michigan State University, 578 \\ S. Shaw Lane, East Lansing, MI 48824, dapro@ chemistry.msu.edu
}

\section{Table of Contents}

Complete set of ODEs

Runge-Kutta boundary conditions

Figure S-1 Potential profile of NPSV

Figure S-2 Residual error from NPSV/GSR of Mb

Figure S-3 Complete depiction of the simplified kinetic model

Figure S-4 Comparison of complete and simplified models

Figure S-5 Determination of $k_{\mathrm{lim}}$ and $k_{\mathrm{el}}^{*}$ of MG at high concentrations

Figure S-6 Concentration dependence of $k_{\text {lim }}$ values for MG.

Figure S-7 Determination of bimolecular rates S-6

Figure S-8 Mediator in determining the minimum NPSV pulse duration S-6 


\section{Complete set of ODEs for a system with a single mediator and analyte pair.}

$$
\begin{gathered}
\frac{\mathrm{d} C_{\mathrm{Ox}}^{\mathrm{M}}}{\mathrm{d} t}=-k_{\mathrm{f}, \mathrm{sol}} C_{\mathrm{Ox}}^{\mathrm{M}} C_{\mathrm{Rd}}^{\mathrm{A}}+k_{\mathrm{r}, \mathrm{sol}} C_{\mathrm{Rd}}^{\mathrm{M}} C_{\mathrm{Ox}}^{\mathrm{A}}-k_{\mathrm{oon}} C_{\mathrm{Ox}}^{\mathrm{M}}+k_{\mathrm{off}} \Gamma_{\mathrm{Ox}}^{\mathrm{M}} \\
\frac{\mathrm{d} \Gamma_{\mathrm{Ox}}^{\mathrm{M}}}{\mathrm{d} t}=k_{\mathrm{on}} C_{\mathrm{Ox}}^{\mathrm{M}}-k_{\mathrm{off}} \Gamma_{\mathrm{Ox}}^{\mathrm{M}}+k_{\mathrm{f}, \mathrm{el}} \Gamma_{\mathrm{Rd}}^{\mathrm{M}}-k_{\mathrm{r}, \mathrm{el}} \Gamma_{\mathrm{Ox}}^{\mathrm{M}} \\
\frac{\mathrm{d} C_{\mathrm{Rd}}^{\mathrm{M}}}{\mathrm{d} t}=k_{\mathrm{f}, \mathrm{sol}} C_{\mathrm{Ox}}^{\mathrm{M}} C_{\mathrm{Rd}}^{\mathrm{A}}-k_{\mathrm{r}, \mathrm{sol}} C_{\mathrm{Rd}}^{\mathrm{M}} C_{\mathrm{Ox}}^{\mathrm{A}}-k_{\mathrm{on}} C_{\mathrm{Rd}}^{\mathrm{M}}+k_{\mathrm{off}} \Gamma_{\mathrm{Rd}}^{\mathrm{M}} \\
\frac{\mathrm{d} \Gamma_{\mathrm{Rd}}^{\mathrm{M}}}{\mathrm{d} t}=k_{\mathrm{on}} C_{\mathrm{Rd}}^{\mathrm{M}}-k_{\mathrm{off}} \Gamma_{\mathrm{Rd}}^{\mathrm{M}}-k_{\mathrm{f}, \mathrm{el}} \Gamma_{\mathrm{Rd}}^{\mathrm{M}}+k_{\mathrm{r}, \mathrm{el}} \Gamma_{\mathrm{Ox}}^{\mathrm{M}} \\
\mathrm{d} t \\
=k_{\mathrm{f}, \mathrm{sol}} C_{\mathrm{Ox}}^{\mathrm{M}} C_{\mathrm{Rd}}^{\mathrm{A}}-k_{\mathrm{r}, \mathrm{sol}} C_{\mathrm{Rd}}^{\mathrm{M}} C_{\mathrm{Ox}}^{\mathrm{A}}-k_{\mathrm{on}} C_{\mathrm{Ox}}^{\mathrm{A}}+k_{\mathrm{off}} \Gamma_{\mathrm{Ox}}^{\mathrm{A}} \\
\frac{\mathrm{d} \Gamma_{\mathrm{Ox}}^{\mathrm{A}}}{\mathrm{d} t}=k_{\mathrm{on}} C_{\mathrm{Ox}}^{\mathrm{A}}-k_{\mathrm{off}} \Gamma_{\mathrm{Ox}}^{\mathrm{A}}+k_{\mathrm{f}, \mathrm{el}} \Gamma_{\mathrm{Rd}}^{\mathrm{A}}-k_{\mathrm{r}, \mathrm{el}} \Gamma_{\mathrm{Ox}}^{\mathrm{A}} \\
\frac{\mathrm{d} C_{\mathrm{Rd}}^{\mathrm{A}}}{\mathrm{d} t}=-k_{\mathrm{f}, \mathrm{sol}} C_{\mathrm{Ox}}^{\mathrm{M}} C_{\mathrm{Rd}}^{\mathrm{A}}+k_{\mathrm{r}, \mathrm{sol}} C_{\mathrm{Rd}}^{\mathrm{M}} C_{\mathrm{Ox}}^{\mathrm{A}}-k_{\mathrm{on}} C_{\mathrm{Rd}}^{\mathrm{A}}+k_{\mathrm{off}} \Gamma_{\mathrm{Rd}}^{\mathrm{A}} \\
\frac{\mathrm{d} \Gamma_{\mathrm{Rd}}^{\mathrm{A}}}{\mathrm{d} t}=k_{\mathrm{on}} C_{\mathrm{Rd}}^{\mathrm{A}}-k_{\mathrm{off}} \Gamma_{\mathrm{Rd}}^{\mathrm{A}}-k_{\mathrm{f}, \mathrm{el}} \Gamma_{\mathrm{Rd}}^{\mathrm{A}}+k_{\mathrm{r}, \mathrm{el}} \Gamma_{\mathrm{Ox}}^{\mathrm{A}}
\end{gathered}
$$

Runge-Kutta boundary conditions: The concentration $C$ (or $\Gamma$ ) of any component in any phase was allowed to rise or fall over the complete step $\Delta t$ to maintain relative change $\Delta_{\Delta t} C=\frac{C_{t+\Delta t}-C_{t}}{C_{t}}$ within the limits of $0.5 \leq \Delta_{\Delta t} C \leq 5$. Relative changes over intermediate sub-steps of Runge-Kutta integration were afforded wider boundary conditions of $0.8 \leq \Delta_{\Delta t} C \leq 100$. If the boundary conditions were violated over either the intermediate or total step, $\Delta t$ was iteratively reduced by $90 \%$ and the simulation step restarted until restrictions were met. If the boundary conditions in the preceding step were satisfied, the next $\Delta t$ was increased by $25 \%$. 


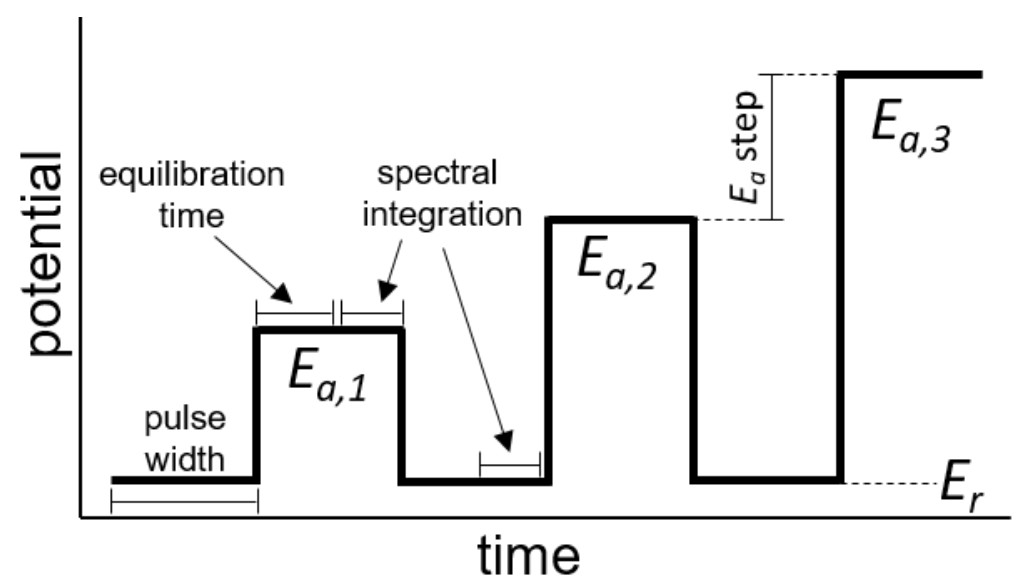

Figure S-1. Potential profile of NPSV. $E_{a}$ alternates between a constant reference, $E_{r}$, and variable applied, $E_{a, i}$, potentials over successive cycles.

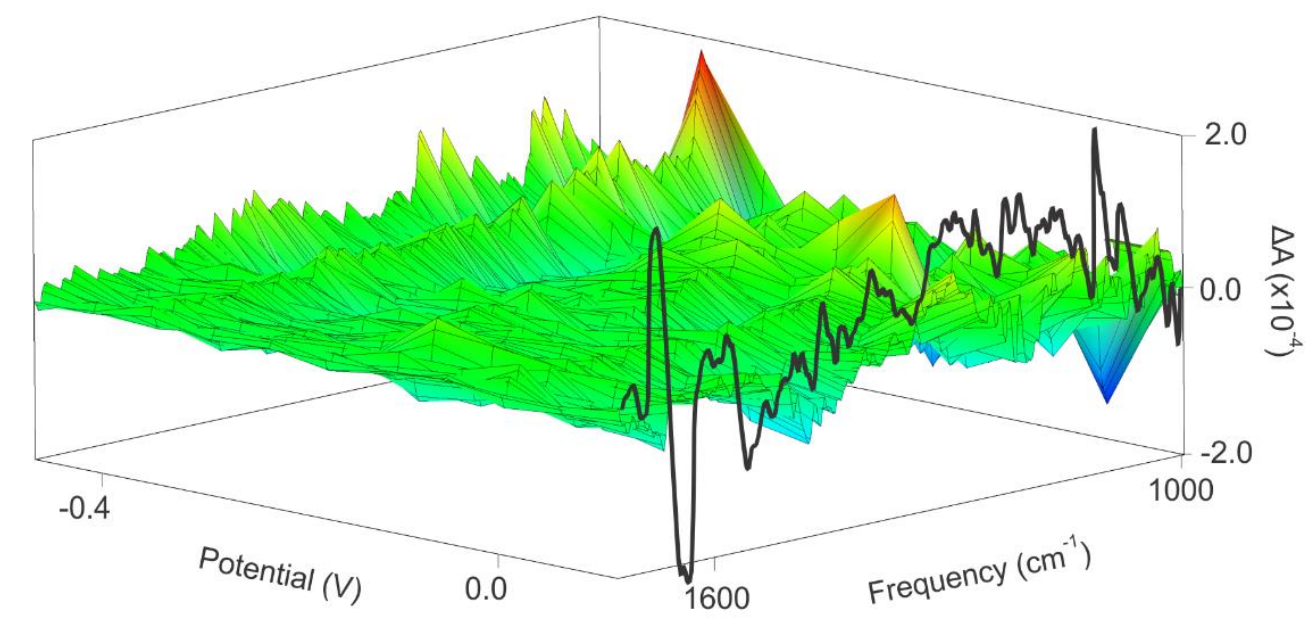

Figure S-2. Residual error from NPSV/GSR of Mb. The full occupancy spectrum of Mb is shown for reference. 

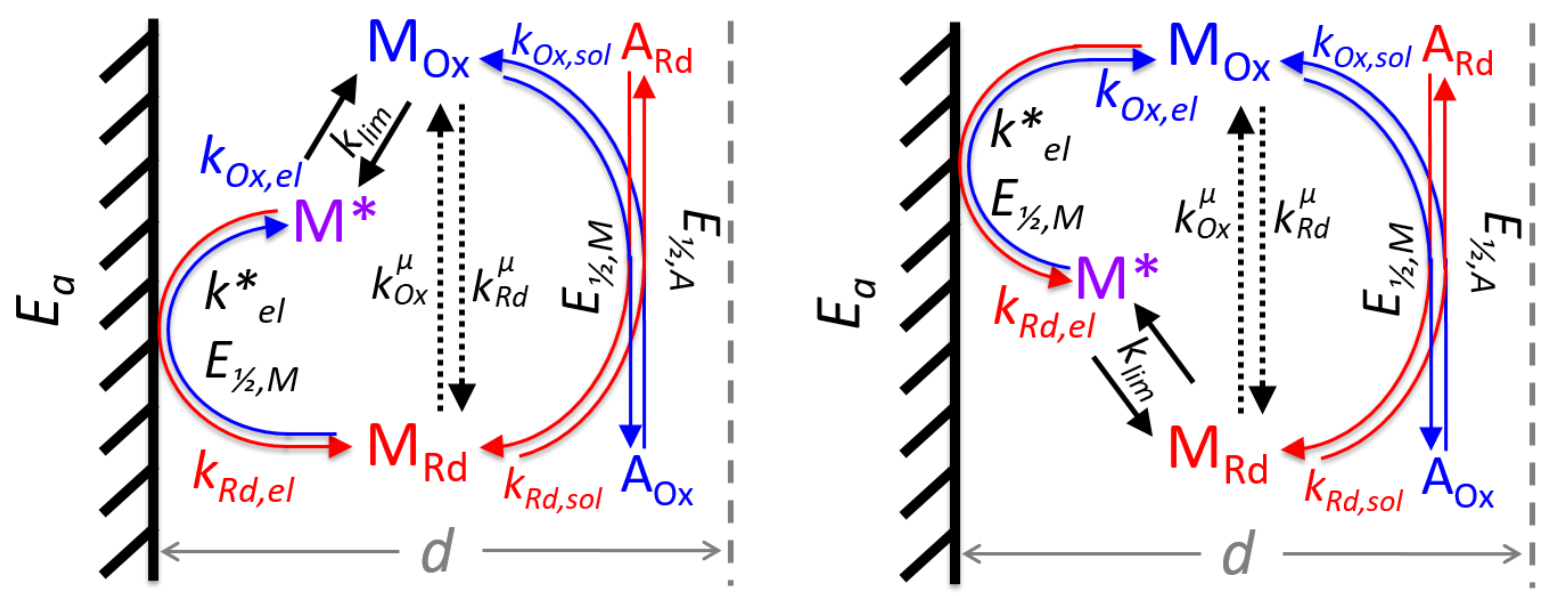

Figure S-3. Complete depiction of the reduced kinetic model showing how $\mathrm{M}^{*}$ can represent $\mathrm{M}_{\mathrm{el}, \mathrm{Ox}}$ or $\mathrm{M}_{\mathrm{el}, \mathrm{Rd}}$ and $k_{\mathrm{lim}}$ is the rate constant of the rate limiting step between $\mathrm{M}^{*}$ and either $\mathrm{M}_{\mathrm{Ox}}$ or $\mathrm{M}_{\mathrm{Rd}}$.

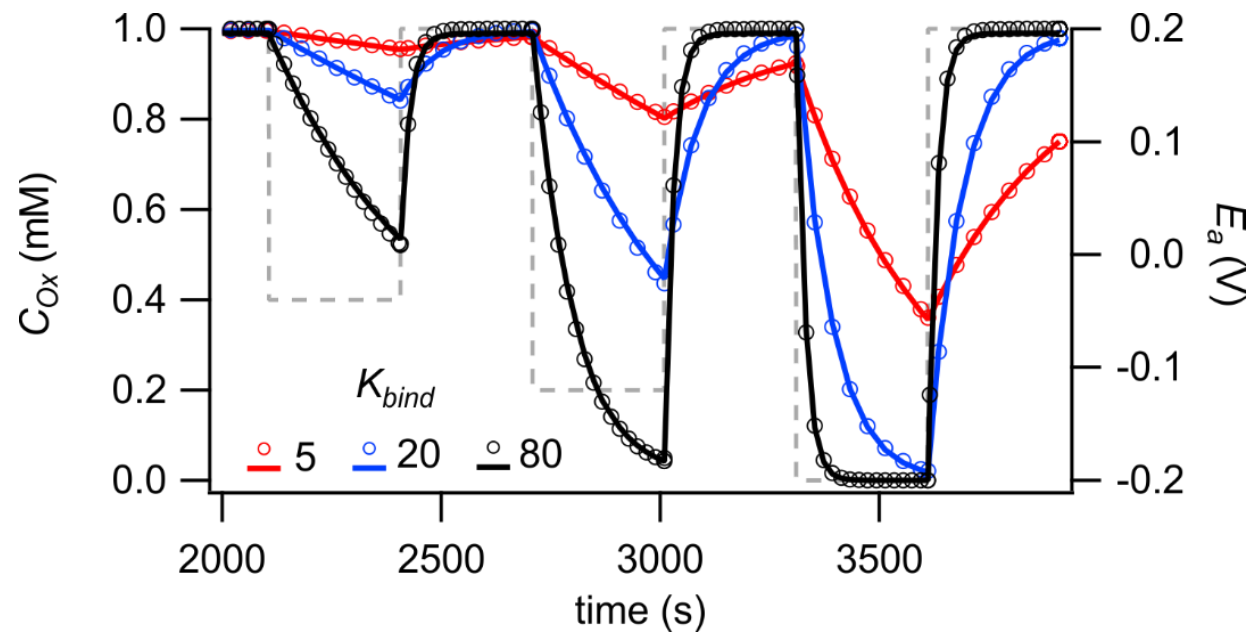

Figure S-4. Comparison of NPV concentration profiles of a single analyte calculated by the complete and reduced models (Fig.

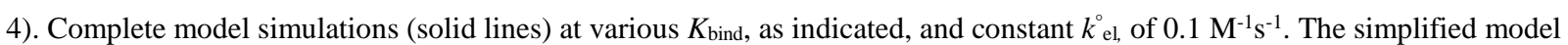
(open circles) used same parameters in the empirical relationship: $k^{*}$ el $=k_{\text {el }}^{\circ} \mathrm{x}\left(K_{\text {bind }}\right) \mathrm{x} h$ where $k_{\mathrm{el}}^{\circ}=0.1 \mathrm{M}^{-1} \mathrm{~s}^{-1}$, the layer thickness $(h)$ was $12 \mu \mathrm{m}$, and $k \lim =k_{\text {on. }}$. 

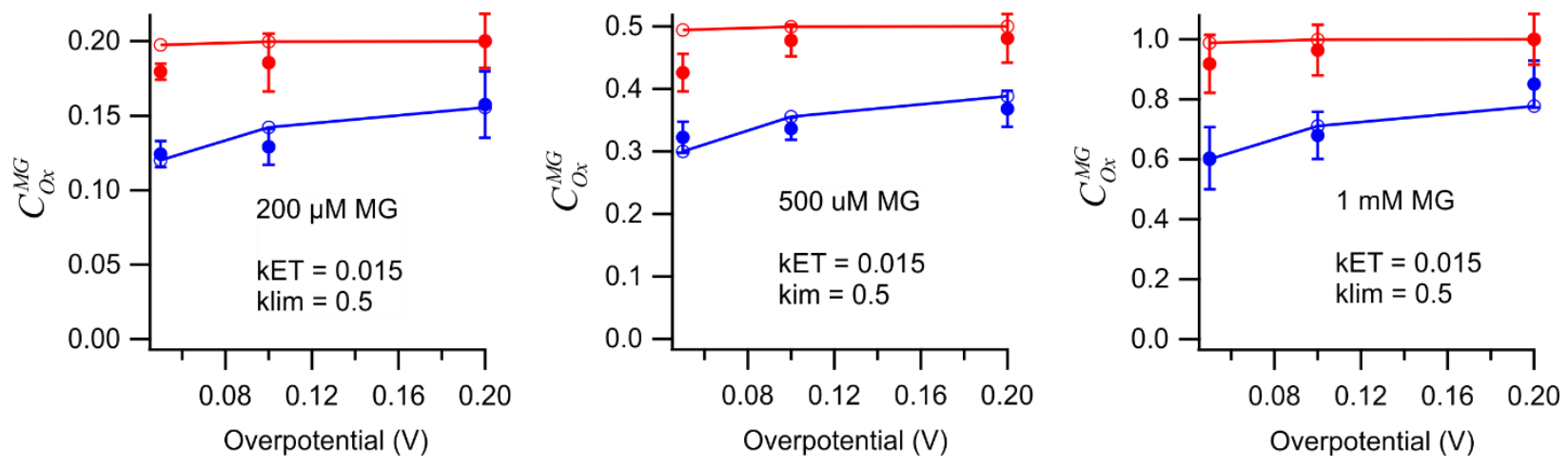

Figure S-5. Determination of $k_{\mathrm{lim}}$ and $k_{\mathrm{el}}^{*}$ of MG at high concentrations. MG samples were prepared in $25 \mathrm{mM}$ Tris, pD 7.0, 0.5

$\mathrm{M} \mathrm{KCl}$ and transferred to the OTTLE cell for FTIR measurements. MG samples were exposed to $50 \mathrm{mV}, 100 \mathrm{mV}$, and $200 \mathrm{mV}$ overpotentials over $15 \mathrm{~s}$ (blue) and $30 \mathrm{~s}$ (red) steps. IR spectra were integrated for $15 \mathrm{~s}$. The intensity at $1603 \mathrm{~cm}^{-1}$ was normalized to the intensity of the completely oxidized sample at the same frequency and then multiplied by the concentration of the sample. Experimental data $(\bullet, \bullet)$ were simulated $(-\bullet,-\bullet)$ by adjusting the values of $k_{\lim }$ and $k_{\mathrm{el}}^{*}$ to find the best fit.

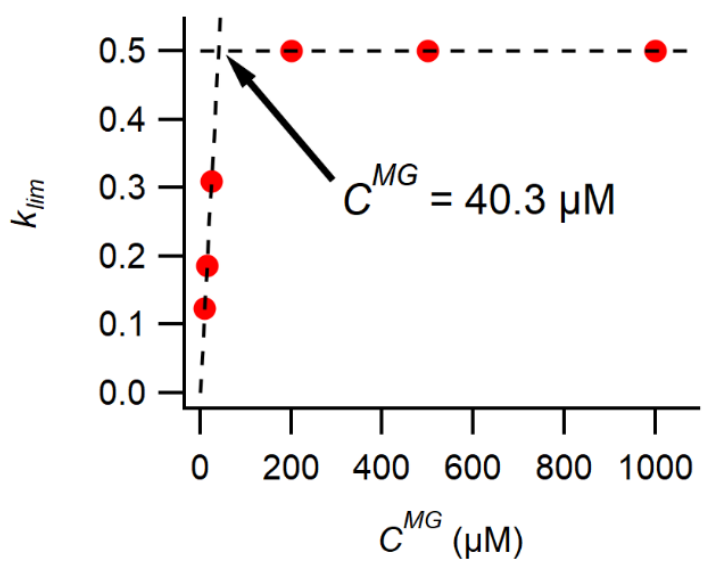

Figure S-6. $k$ lim values for MG determined at various concentrations. $k$ lim follows a linear profile at low mediator concentrations and reaches a constant value of 0.5 at $40.3 \mu \mathrm{M}$ MG. 

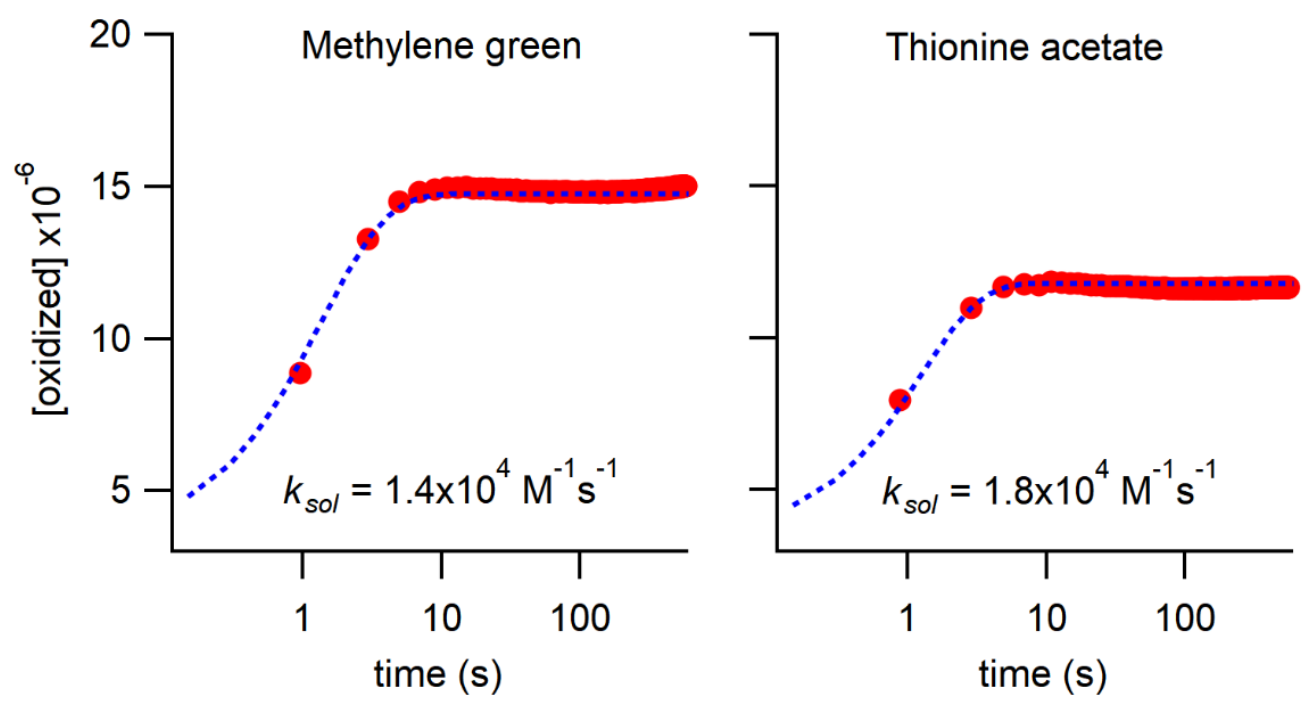

Figure S-7. Determination of homogenous bimolecular rates of reactions between mediators and Mb. Optical changes at characteristic wavelengths were recorded in $2 \mathrm{~s}$ intervals as a 0.5 molar equivalent of $\mathrm{Mb}$ was added to the reduced MG or TA. Absorbance profiles were taken from $611 \mathrm{~nm}$ and $598 \mathrm{~nm}$ for MG and TA, respectively, and converted to concentrations using their corresponding $\varepsilon$. The quantitative model was used to find the best $k_{\text {sol }}$ value to simulate $(. . . .$.$) the experimental (\bullet)$ concentration profiles.
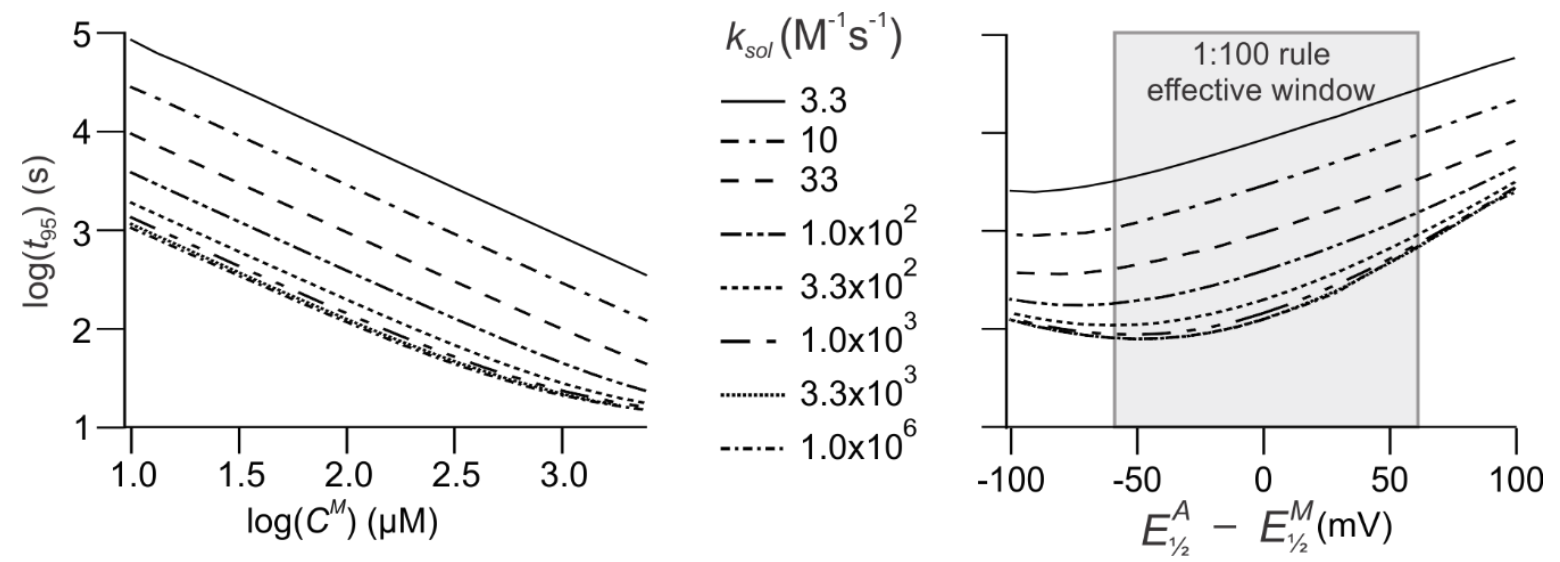

Figure S-8. The role of mediator in determining the minimum NPSV pulse duration. The minimal pulse duration ( $\left.t_{95}\right)$ is calculated for of an arbitrary $1 \mathrm{mM}$ analyte with $k_{\text {el }}^{*}$ of 0.1 and $k_{\lim }$ of 0.4 following a potential step of $E_{\mathrm{a}}=E_{1 / 2}^{\mathrm{A}}+100 \mathrm{mV}$ in the presence of a mediator with given bimolecular rate constant $k_{\text {sol. }}$. Left: the effect of mediator concentration at $E_{1 / 2}^{\mathrm{A}}=E_{1 / 2}^{\mathrm{M}}$. Right: the effect of $E_{1 / 2}^{\mathrm{M}}$ relative to $E_{1 / 2}^{\mathrm{A}}$ for a constant mediator concentration of $100 \mu \mathrm{M}$. 